\title{
Evaluation of bispectrum in the wave number domain based on multi-point measurements
}

\author{
Y. Narita ${ }^{1}$, K.-H. Glassmeier ${ }^{1}$, P. M. E. Décréau ${ }^{2}$, T. Hada $^{3}$, U. Motschmann ${ }^{4}$, and Y. Nariyuki ${ }^{5}$ \\ ${ }^{1}$ Institut für Geophysik und extraterrestrische Physik, Mendelssohnstr. 3, 38106 Braunschweig, Germany \\ ${ }^{2}$ LPCE/CNRS and Université d'Orléans, Orléans, France \\ ${ }^{3}$ Department of Earth System Science and Technology, Kyushu University, 816-8580, Kasuga, Fukuoka, Japan \\ ${ }^{4}$ Institut für Theoretische Physik, Mendelssohnstr. 3, 38106 Braunschweig, Germany \\ ${ }^{5}$ Department of electrical engineering, Kochi National College of Technology, Monobe-Otsu 200-1, Nankoku, Kochi \\ 783-8508, Japan
}

Received: 30 July 2008 - Revised: 28 August 2008 - Accepted: 21 October 2008 - Published: 24 October 2008

\begin{abstract}
We present an estimator of the bispectrum, a measure of three-wave couplings. It is evaluated directly in the wave number domain using a limited number of detectors. The ability of the bispectrum estimator is examined numerically and then it is applied to fluctuations of magnetic field and electron density in the terrestrial foreshock region observed by the four Cluster spacecraft, which indicates the presence of a three-wave coupling in space plasma.
\end{abstract}

Keywords. Interplanetary physics (Plasma waves and turbulence) - Space plasma physics (Experimental and mathematical techniques; Wave-wave interactions)

\section{Introduction}

Higher order statistics is of great importance in studying nonlinear processes of turbulence. They reflect the shape of probability distribution functions of fluctuations. Theoretical treatments of turbulent fields often assume the Gaussian or almost-Gaussian distribution (called the quasi-normal approximation) to solve the closure problem of turbulence. In the frame of weak turbulence the breakdown of the Gaussian distribution can be interpreted as a sign of phase coherence, e.g. three-wave or four-wave couplings. Estimating the bispectrum provides the very means to measure the magnitude of three-wave couplings (see review of Dudok de Wit, 2003). In laboratory and space plasma physics the method of the bispectrum has been wodely applied: in ionospheric radio emission (Lagoutte et al., 1989); in laboratory plasmas (Kim and Powers, 1979; Ritz et al., 1989; Brochard et al., 2006); and

Correspondence to: Y. Narita

(y.narita@tu-bs.de) upstream waves of the Earth's quasi-parallel bow shock (Dudok de Wit and Krasnosel'skikh, 1995). The bispectrum investigations have been made solely in the frequency domain, as one uses single point measurements and therefore time series data. Also, Taylor's hypothesis is often applied in a flowing medium and the bispectra in the frequency domain reflect that in the wave number domain. However, the wave-wave couplings should be examined in the wave number domain, too, which motivates us to estimate the bispectrum directly in the wave number domain using only a limited number of measurement points. For example, with Taylor's hypothesis one cannot measure the wave number perpendicular to the flow. Here we present an estimator of the bispectrum. The ability of the estimator is examined numerically and then it is applied to the fluctuations in the Earth foreshock region. While wave-wave interactions are extensively studied with simulation data Nariyuki and Hada (2006), to the authors' knowledge it is the first time that the wave-wave coupling is identified in the wave number domain with experimental space plasma data.

\section{Estimator of bispectrum}

Suppose a scalar field $A(\boldsymbol{r}, t)$ is measured by an $N$ point sensor array. The scalar field can be not only density or temperature of the medium but also any components of magnetic fields or velocity fields. We determine the state vector in the frequency domain from the measurement as

$\boldsymbol{A}(\omega)=\left(A_{1}(\omega), A_{2}(\omega), \cdots, A_{N}(\omega)\right)$,

where $A_{i}(\omega)$ denotes the Fourier transform of the scalar field measured by the $i$-th sensor into the frequency domain. The

Published by Copernicus Publications on behalf of the European Geosciences Union. 
state vector is then projected into a scalar $a$ with a suitable weight vector $\boldsymbol{W}$ as

$a(\omega, \boldsymbol{k})=\boldsymbol{W}^{\dagger}(\omega, \boldsymbol{k}) \cdot \boldsymbol{A}(\omega)$,

where the dagger $\dagger$ denotes the Hermitian conjugate. The scalar $a$ represents the amplitude of a plane wave with frequency $\omega$ and wave vector $\boldsymbol{k}$, retaining phase information. We use Capon's weight vector (Capon, 1969)

$\boldsymbol{W}(\omega)=\frac{\mathbf{R}^{-1}(\omega) \boldsymbol{H}(\boldsymbol{k})}{\boldsymbol{H}^{\dagger}(\boldsymbol{k}) \mathbf{R}^{-1}(\omega) \boldsymbol{H}(\boldsymbol{k})}$,

where $\boldsymbol{H}(\boldsymbol{k})$ is called the steering vector,

$\boldsymbol{H}(\boldsymbol{k})=\left(\exp \left(i \boldsymbol{k} \cdot \boldsymbol{r}_{1}\right), \cdots, \exp \left(i \boldsymbol{k} \cdot \boldsymbol{r}_{N}\right)\right)$

and $\mathbf{R}(\omega)$ is the covariance matrix of the state vector,

$\mathbf{R}(\omega)=\left\langle\boldsymbol{A}(\omega) \boldsymbol{A}^{\dagger}(\omega)\right\rangle$.

The angular bracket $\langle\cdot \cdot\rangle$ denotes the ensemble average. In practice the averaging is made over time-subintervals. Capon's weight vector stems from an optimization procedure under a certain constraint. Consider to minimize the mean square amplitude of the field $\left\langle|a|^{2}\right\rangle$ (which is the wave energy) under constraint that the weight vector serves as a unit gain, that is

minimize $\left\langle|a|^{2}\right\rangle \quad$ subject to $\quad \boldsymbol{W}^{\dagger} \cdot \boldsymbol{H}=1$.

This problem can be analytically solved by the method of Lagrangian multiplier, yielding the weight vector in Eq. (3). See Haykin (1991) for its derivation. In principle one could also find another weight vector in such a way that the third order moment is minimized under the unit gain constraint, but the analytical treatment becomes more elaborate. We choose here Capon's weight vector for the sake of simplicity. Capon's method can be generalized to vector fields (magnetic fields, velocities) to determine the wave energy distribution in the frequency and wave number domain, referred to as the wave telescope (Motschmann et al., 1996; Glassmeier et al., 2001). The k-filtering (Pinçon and Lefeuvre, 1991) is another approach to estimate the energy distribution in the wave number domain.

The projection using the weight vector furthermore enables one to determine higher order moments of the fluctuations. The third order moment, for example, is expressed as $\left\langle a a^{\prime}\left(a^{\prime \prime}\right)^{*}\right\rangle$, where the asterisk ${ }^{*}$ denotes the complex conjugate. The prime and the double prime denote that the wave amplitudes are estimated at different frequencies and wave numbers. It is worthwhile to note that the third order moment becomes non-zero only if the three wave fields $a, a^{\prime}$, and $a^{\prime \prime}$ are in resonance, satisfying the frequency and the wave number matching, $\omega+\omega^{\prime}=\omega^{\prime \prime}$ and $\boldsymbol{k}+\boldsymbol{k}^{\prime}=\boldsymbol{k}^{\prime \prime}$, otherwise the third order moment vanishes because of random phase mixing. The bispectrum in the wave number domain is obtained from the third order moment as

$F\left(\boldsymbol{k}, \boldsymbol{k}^{\prime}\right)=\frac{1}{T \Delta \boldsymbol{k} \Delta \boldsymbol{k}^{\prime}} \int\left\langle a a^{\prime}\left(a^{\prime \prime}\right)^{*}\right\rangle d \omega d \omega^{\prime}$, where $\Delta \boldsymbol{k}$ and $\Delta \boldsymbol{k}^{\prime}$ denote the grid size of the wave number space and $T$ the time length of the measurement. The factor $1 / T \Delta \boldsymbol{k} \Delta \boldsymbol{k}^{\prime}$ sets the units of the bispectrum properly. Since the weight vector is dimensionless, the units of the estimated amplitude is the same as the Fourier transform of the field into the frequency domain, whereas the units of the bispectrum are the density of the cubic amplitude in the wave number domain, (amplitude) $)^{3} /$ (wave number) $^{2}$.

\section{Applications}

\subsection{Numerical test}

The quality of the bispectrum estimator is examined with a numerical test. We generate waves in the magnetic field and the number density under a three-wave resonance in onedimension. This type of wave-wave coupling is typical for the parametric instabilities of large amplitude Alfvén waves (Longtin and Sonnerup, 1986; Terasawa et al., 1986; Wong and Goldstein, 1986). The generated fluctuations are sampled at four discrete spatial points in the system. We then evaluate the bispectrum based on the time series at the four points to identify the wave-wave coupling in the wave number domain.

The first component of the wave fields is a magnetic field oscillation, and the second component a density oscillation. Their frequencies and wave numbers are randomly chosen $\left(\omega_{1}=0.39 \mathrm{rad} / \mathrm{s}, \omega_{2}=0.21 \mathrm{rad} / \mathrm{s}, k_{1}=0.0059 \mathrm{~km}^{-1}\right.$, $\left.k_{2}=0.0104 \mathrm{~km}^{-1}\right)$. The third wave component is a magnetic field oscillation satisfying the resonance condition $\omega_{1}+\omega_{2}=\omega_{3}, k_{1}+k_{2}=k_{3}$. The amplitudes are $10 \mathrm{nT}, 1 \mathrm{~cm}^{-3}$, $9 \mathrm{nT}$ for the three wave fields, respectively. These fluctuations are superposed on the background fields ( $23 \mathrm{nT}$ for the magnetic field and $5 \mathrm{~cm}^{-3}$ for the density). In addition we put broadband wave activity with phases random to one another. The fluctuations are sampled at four separate positions at distance $100 \mathrm{~km}, 180 \mathrm{~km}, 290 \mathrm{~km}$, and $375 \mathrm{~km}$. Figure 1 displays the time series of the magnetic field and the density sampled at the sensor 1 .

The bispectrum is then estimated from the four-point measurements of the magnetic field and the density fluctuations. First we compute the third order moment

$C\left(\omega, \omega^{\prime}, k, k^{\prime}\right)=\left\langle b(\omega, k) n\left(\omega^{\prime}, k^{\prime}\right) b^{*}\left(\omega+\omega^{\prime}, k+k^{\prime}\right)\right\rangle$,

where $b$ and $n$ denote the projection of the state vector of the magnetic field $\boldsymbol{B}(\omega)$ and that of the density $\boldsymbol{N}\left(\omega^{\prime}\right)$,

$$
\begin{aligned}
b(\omega, k) & =\boldsymbol{W}_{b}^{\dagger}(\omega, k) \cdot \boldsymbol{B}(\omega) \\
n\left(\omega^{\prime}, k^{\prime}\right) & =\boldsymbol{W}_{n}^{\dagger}\left(\omega^{\prime}, k^{\prime}\right) \cdot \boldsymbol{N}\left(\omega^{\prime}\right) .
\end{aligned}
$$

We use different weight vectors for the magnetic field and the density,

$$
\boldsymbol{W}_{b}(\omega, k)=\frac{\mathbf{R}_{b}^{-1}(\omega) \boldsymbol{H}(k)}{\boldsymbol{H}^{\dagger}(k) \mathbf{R}_{b}^{-1}(\omega) \boldsymbol{H}(k)}
$$



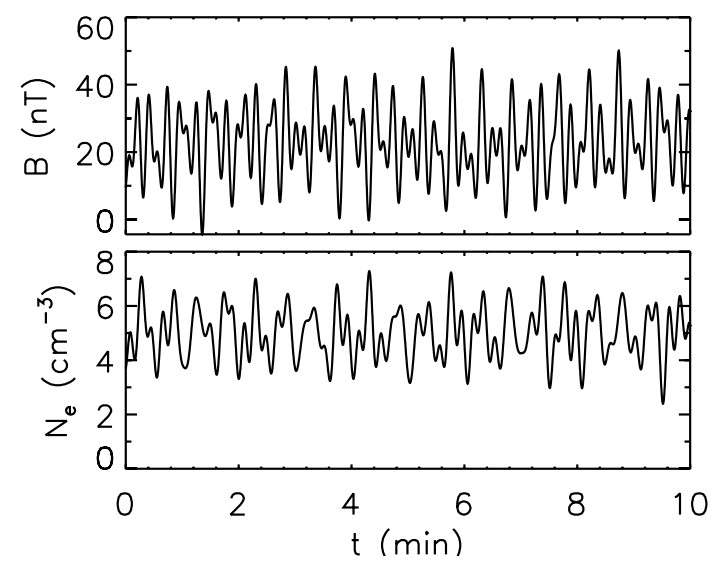

Fig. 1. Time series of artificial magnetic field and density fluctuation at the virtual sensor 1 .

$\boldsymbol{W}_{n}\left(\omega^{\prime}, k^{\prime}\right)=\frac{\mathbf{R}_{n}^{-1}\left(\omega^{\prime}\right) \boldsymbol{H}\left(k^{\prime}\right)}{\boldsymbol{H}^{\dagger}\left(k^{\prime}\right) \mathbf{R}_{n}^{-1}\left(\omega^{\prime}\right) \boldsymbol{H}\left(k^{\prime}\right)}$,

where $R_{b}(\omega)=\left\langle\boldsymbol{B}(\omega) \boldsymbol{B}^{\dagger}(\omega)\right\rangle$ and $R_{n}\left(\omega^{\prime}\right)=\left\langle\boldsymbol{N}\left(\omega^{\prime}\right) \boldsymbol{N}^{\dagger}\left(\omega^{\prime}\right)\right\rangle$. The bispectrum is obtained by integrating the third order moment over the two frequency domains,

$F\left(k, k^{\prime}\right)=\frac{1}{T \Delta k \Delta k^{\prime}} \int C\left(\omega, \omega^{\prime}, k, k^{\prime}\right) d \omega d \omega^{\prime}$.

We use a constant grid in the wave number domain $\Delta k=\Delta k^{\prime}=5 \times 10^{-3} \mathrm{~km}^{-1}$ (grid size is limited by computational resource). Figure 2 displays the bispectrum for the numerical data set. The bispectrum exhibits a dominant peak just on the resonance condition (the dotted line), $k+k^{\prime}=0.0163 \mathrm{~km}^{-1}$, which confirms the resonance pair of wave numbers. Our bispectral analysis can identify the location of the wave-wave coupling in the wave number domain, although the broadband wave activity is present.

\subsection{Cluster observation in foreshock}

The bispectrum estimator is then applied to the fluctuation in the Earth foreshock region. The foreshock is located upstream of the Earth bow shock, typically beyond $20 R_{E}$ (Earth radius, ca. $6400 \mathrm{~km}$ ) ahead of the Earth, where the interplanetary magnetic field contacts the bow shock at angles quasi-parallel to the shock normal direction. The foreshock is one of the active regions of large amplitude Alfvén waves, since the backstreaming ions from the shock against the solar wind flow provides an energy to excite the Alfvén waves. It is likely that the foreshock exhibits wave-wave coupling processes, as identified by Spangler et al. (1997) in the frequency domain. We use the magnetic field measurement (Balogh et al., 2001) and the electron density measurement (Décréau et al., 2001) on board the four Cluster spacecraft (Escoubet et al., 2001). In the time interval 18 February

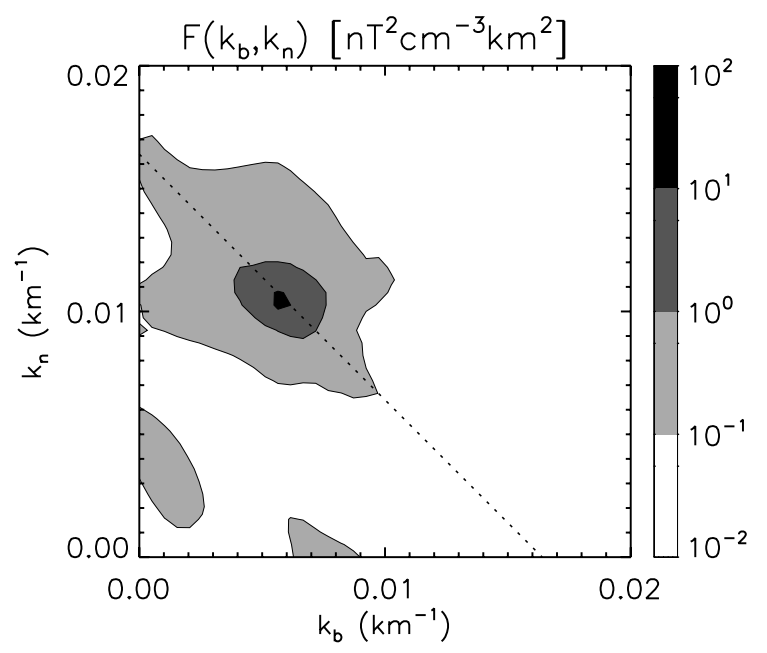

Fig. 2. Bispectrum for the numerical test. The $x$ - and $y$-axes are the wave number of the magnetic field and the density fluctuation, respectively.

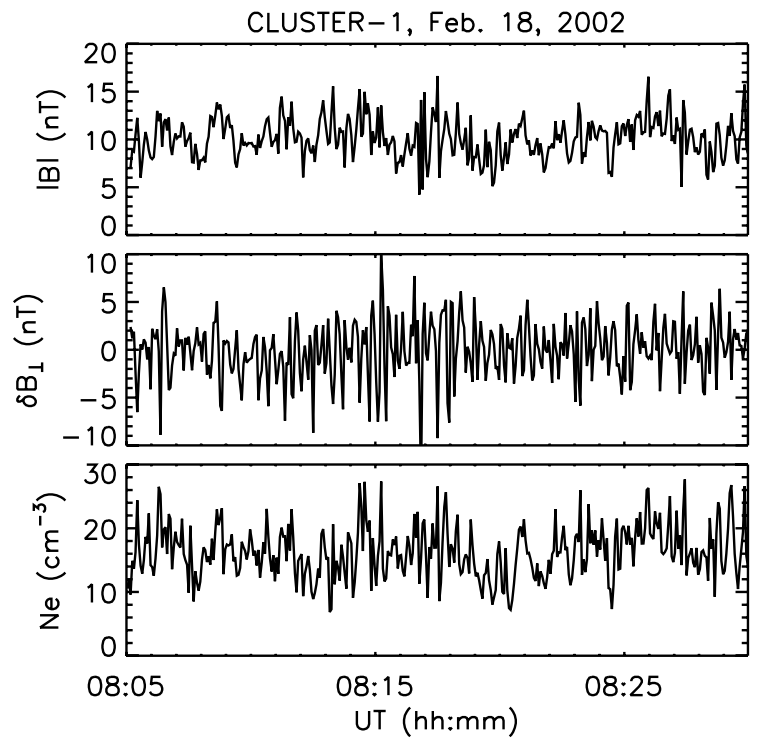

Fig. 3. Time series of the magnetic field magnitude (top), the fluctuation perpendicular to the mean field (middle), and the electron density fluctuation (bottom) obtained by Cluster-1 in the foreshock region.

2002, 18:05-18:35 UT Cluster encountered the foreshock region in its outbound orbit. The spacecraft separation is about $100 \mathrm{~km}$. The background quantities are $10 \mathrm{nT}$ for the magnetic field and $16 \mathrm{~cm}^{-3}$ for the electron density. Figure 3 displays the time series of the magnetic field (magnitude and perpendicular component) and the electron density used in the analysis. 


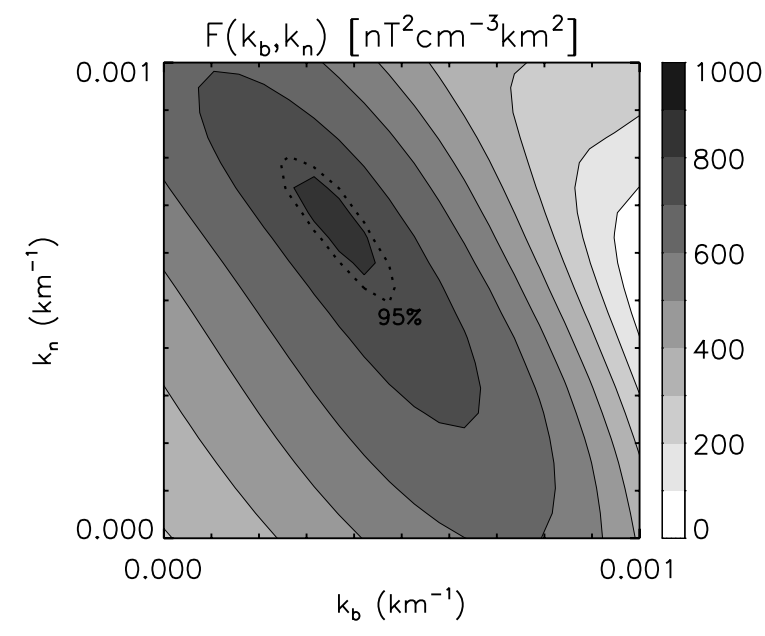

Fig. 4. Bispectrum for the Cluster observation in the foreshock with the same style as Fig. 2. The dotted line denotes the level of 95\% confidence.

The bispectrum is determined with the same procedure as that of the numerical test, with the grid size $5 \times 10^{-5} \mathrm{~km}^{-1}$ (here the grid size is again limited by the computational resource). We use the perpendicular component of the magnetic field and the electron density fluctuation associated with wave vectors parallel to the magnetic field. The result is displayed in Fig. 4. A peak is identified at the pair of wave numbers around $k_{b}=3.5 \times 10^{-4} \mathrm{~km}^{-1}$ and $k_{n}=6.5 \times 10^{-4} \mathrm{~km}^{-1}$ for the magnetic field and the density fluctuations, respectively. Figure 4 also shows the $95 \%$ confidence level about the peak. We assume a normal distribution for the evaluation of the bispectrum and use 16 degrees of freedom in frequency estimates (the number of time sub-windows) and in addition 256 in bispectrum estimates. The distribution of the bispectrum tends to be aligned to a resonance line (straight line with the slope -1, see the dotted line in Fig. 2), but the alignment is slightly steeper than the resonance line.

\section{Conclusions}

The bispectral analysis provides useful information about wave-wave interactions. However, one should be careful in that the bispectum is a necessary but not a sufficient condition for having three-wave interactions. A nonlinearity in the detector, for example, may give rise to a non-zero bispectrum (Walker et al., 2002), or one may have four-wave interactions. Also, the higher order moments may be remnants of wave-wave interactions that took place somewhere upstream and were convected by the flow. However, our method can successfully identify a peak in experimental space plasma data within the limit of the confidence level, which is an indication that the three-wave coupling may really be present in space plasma.
Although the number of measurement points is too small to perform the Fourier transform from the spatial coordinate into wave numbers, it is possible to estimate the higher order moments in the wave number domain. This method is useful even in regions like the solar wind where Taylor's hypothesis generally holds because with this hypothesis one can estimate wave numbers in the flow direction and not perpendicular to the flow. Therefore it is worthwhile performing the bispectral analysis in the wave number domain even in the foreshock or the solar wind. Our method can be used to study the parametric instabilities of large amplitude Alfvén waves, in which a parent Alfvén wave collapses into a sound wave and daughter Alfvén waves (Longtin and Sonnerup, 1986; Terasawa et al., 1986; Wong and Goldstein, 1986) in the regions of foreshock (Spangler et al., 1997) or solar wind (Marsch and Tu, 1997).

Acknowledgements. This work was financially supported by Bundesministerium für Wirtschaft und Technologie and Deutsches Zentrum für Luft- und Raumfahrt, Germany, under contract 50OC0103.

Topical Editor I. A. Daglis thanks one anonymous referee for her/his help in evaluating this paper.

\section{References}

Balogh, A., Carr, C. M., Acuña M. H., et al.: The Cluster magnetic field investigation: overview of in-flight performance and initial results Ann. Geophys, 19, 1207-1217,2001.

Brochard, F., Windisch, T., Grulke, O., and Klinger, T.: Experimental evidence of mode coupling in drife wave intermittent turbulence using a wave nunber bicoherence analysis, Phys. Plasmas, 13, 122305, doi:10.1063/1.2402131, 2006.

Capon, J.: High Resolution Frequency-Wavenumber Spectrum Analysis, Proc. IEEE, 57, 1408-1418, 1969.

Escoubet, C. P., Fehringer, M., and Goldstein, M.: The Cluster mission, Ann. Geophys., 19, 1197-1200, 2001, http://www.ann-geophys.net/19/1197/2001/.

Décréau, P. M. E., Fergeau, P., Krasnoselskikh, V., et al.: Early results from the Whisper instrument on Cluster: an overview, Ann. Geophys., 19, 1241-1258, 2001, http://www.ann-geophys.net/19/1241/2001/.

Dudok de Wit, T. and Krasnosel'skikh, V. V.: Wavelet bicoherence analysis of strong plasma turbulence at the Earth's quasiparallel bow shock, Phys. Plasmas, 2, 4307-4311, 1995.

Dudok de Wit, T.: Numerical schemes for the analysis of turbulence - A tutorial, Space Plasma Simulation, edited by: Büchner, J., Dum, C. T., and Scholer, M., Springer, Berlin, 315-344, 2003.

Glassmeier, K.-H., Motschmann, U., Dunlop, M., et al.: Cluster as a wave telescope - first results from the fluxgate magnetometer, Ann. Geophys., 19, 1439-1447, 2001 (Correction, 21, 1071, 2003).

Haykin, S.: Adaptive filter theory, 2nd ed., Prentice Hall information and system science series, Prentice-Hall Inc., New Jersey, pp. 396-399, 1991.

Kim, Y. C. and Powers, E. J.: Digital bispectral analysis and its applications to nonlinear wave interactions, IEEE Trans. Plasma Sci., PS-7, 120-131, 1979. 
Lagoutte, D., Lefeuvre, F., and Hanasz, J.: Application of bicoherence analysis in study of wave interactions in space plasma, J. Geophys. Res., 94, 435-442, 1989.

Longtin, M. and Sonnerup, B.: Modulational instability of circularly polarized Alfvén waves, J. Geophys. Res., 91, 798-801, 1986.

Marsch, E. and Tu, C.-Y.: Intermittency, non-Gaussian statistics and fractal scaling of MHD fluctuations in the solar wind, Nonlin. Processes Geophys., 4, 101-124, 1997,

http://www.nonlin-processes-geophys.net/4/101/1997/.

Motschmann, U., Woodward, T. I., Glassmeier, K.-H., Southwood, D. J., and Pinçon, J. L.: Wavelength and direction filtering by magnetic measurements at satellite arrays: generalized minimum variance analysis, J. Geophys. Res., 101, 4961-4965, 1996.

Nariyuki, Y. and Hada, T.: Remarks on nonlinear relation among phases and frequencies in modulational instabilities of parallel propagating Alfvn waves, Nonlin. Processes Geophys., 13, 425441, 2006,

http://www.nonlin-processes-geophys.net/13/425/2006/.
Pinçon, J. L. and Lefeuvre, F.: Local characterization of homogeneous turbulence in a space plasma from simultaneous measurement of field components at several points in space, J. Geophys. Res., 96, 1789-1802, 1991.

Ritz, Ch. P., Powers, E. J., and Bengtson, R. D.: Experimental measurement of three-wave coupling and energy cascading, Phys. Fluids B, 1, 153-163, 1989.

Spangler, S. R., Leckband, J. A., and Cairns, I. H.: Observations of the parametric decay instability of nonlinear magnetohydrodynamic waves, Phys. Plasmas, 4, 846-855, 1997.

Terasawa, T., Hoshino, M., Sakai, J.-I., and Hada, T.: Decay instability of finite-amplitude circularly polarized Alfvén waves: A numerical simulation of stimulated brillouin scattering, J. Geophys. Res., 91, 4171-4187, 1986.

Walker, S. N., Balikhin, M. A., Bates, I., and Huff, R.: An investigation into instrumental nonlinear effects, Adv. Space Res., 30, 2815-2820, 2002.

Wong, H. K. and Goldstein, M. L.: Parametric instabilities of circularly polarized Alfvén waves including dispersion, J. Geophys. Res., 91, 5617-5628, 1986. 